Review

\title{
Treatment of myocardial ischaemia-reperfusion injury in patients with ST-segment elevation myocardial infarction: promise, disappointment, and hope
}

\author{
Jingzhou $\mathrm{He}^{1,2, *}$, Nick G Bellenger ${ }^{1,2}$, Andrew J Ludman ${ }^{1,2}$, Angela C Shore ${ }^{1,3}$, \\ W David Strain ${ }^{1,3}$ \\ ${ }^{1}$ Diabetes and Vascular Medicine Research Centre, Institute of Biomedical and Clinical Science and University of Exeter, College of Medicine and \\ Health, Royal Devon \& Exeter Hospital, EX2 5AX Exeter, UK \\ ${ }^{2}$ Department of Cardiology, Royal Devon \& Exeter Hospital, EX2 5AX Exeter, UK \\ ${ }^{3}$ NIHR Exeter Clinical Research Facility, Royal Devon \& Exeter NHS Foundation Trust and University of Exeter, College of Medicine and Health, \\ RILD building, Royal Devon \& Exeter Hospital, EX2 5DW Exeter, UK \\ *Correspondence: Jingzhou.he@nhs.net (Jingzhou He) \\ Academic Editors: Filippos Triposkiadis, Massimo Volpe, Grigorios Korosoglou and Matteo Cameli \\ Submitted: 31 August 2021 Revised: 20 December 2021 Accepted: 22 December 2021 Published: 17 January 2022
}

\begin{abstract}
Acute myocardial infarction (AMI) is a major cause of morbidity and mortality worldwide. Timely reperfusion with primary percutaneous coronary intervention (PPCI) remains the gold standard in patients presenting with ST-segment elevation myocardial infarction (STEMI), limiting infarct size, preserving left ventricular ejection fraction (LVEF), and improving clinical outcomes. Despite this, a significant proportion of STEMI patients develop post-infarct heart failure. We review the current understanding and up-to-date evidence base for therapeutic intervention of ischaemia-reperfusion injury (IRI), a combination of myocardial ischaemia secondary to acute coronary occlusion and reperfusion injury leading to further myocardial injury and cell death. Multiple treatment modalities have been shown to be cardioprotective and reduce IRI in experimental animal models. Recent phase II/III randomised controlled trials (RCT) have assessed multiple cardioprotective strategies ranging from ischaemic conditioning, therapeutic hypothermia and hyperoxaemia to pharmacological therapies. While several therapies have been shown to reduce infarct size in animal models or proof-of-concept studies, many larger scale trial results have proven inconsistent and disappointing. Hard clinical outcomes remain elusive. We discuss potential reasons for the difficulties in translation to clinical practice.
\end{abstract}

Keywords: Acute myocardial infarction; Coronary microvascular dysfunction; Ischaemia reperfusion injury; Cardioprotection; Ischaemic conditioning

\section{Introduction}

Ischaemic heart disease (IHD) is the leading cause of death worldwide with around 1.8 million deaths per year in Europe alone, accounting for $20 \%$ of all deaths [1]. Primary percutaneous coronary intervention (PPCI) is the gold standard reperfusion strategy for patients presenting with an acute ST-segment elevation myocardial infarction (STEMI) within $12 \mathrm{~h}$ of symptom onset. The end goal is to ensure adequate blood supply to ischaemic but viable myocardium in order to reduce infarct size, preserve left ventricular (LV) function, and reduce mortality [2,3].

Up to $95 \%$ of occluded coronary vessels are re-opened in this manner [4]. However, despite prompt reperfusion and optimal medical therapy, up to $22 \%$ of individuals will experience a prolonged or new hospitalisation for heart failure within a year, with up to $7 \%$ mortality at 1 year [5]. One of the main determinants of mortality, heart failure, and arrhythmia after acute myocardial infarction is infarct size [6-9]. A meta-analysis by Stone et al. [10] of 1889 patients assessed after STEMI by cardiac magnetic resonance imaging (CMRI) and 743 by single-photon emission com- puterised tomography (SPECT) found an independent association between infarct size within 1 month, and subsequent mortality and hospitalisation for heart failure at 1 year.

While timely reperfusion with PPCI is essential to salvage myocardium, the process of restoring coronary blood flow can induce myocardial injury and death of cells that were only reversibly injured during the preceding ischaemic episode $[11,12]$. The ongoing damage to myocardial tissue and coronary microvasculature that occurs after blood supply is restored is a complex multifactorial process that is estimated to account for up to $50 \%$ of the final infarct size [11]. It is therefore the summative effects of both myocardial ischaemia and lethal reperfusion injury that contribute to the final infarct size and clinical outcomes.

There is no doubt regarding the evidence and efficacy for PPCI in reducing infarct size. However, cardioprotective strategies for prevention of lethal reperfusion injury and coronary microvascular dysfunction (CMD) remain limited. This mini review aims to cover the basic pathophysiology of ischaemia-reperfusion injury (IRI) and summarise the attempts made to translate cardioprotective strategies into the clinical setting. 


\section{ISCHAEMIA}

\section{Mitochondrial depolarisation, ATP depletion, $\mathrm{Na}^{+}$ and $\mathrm{Ca}^{2+}$ overload, acidosis, ROS}

REPERFUSION

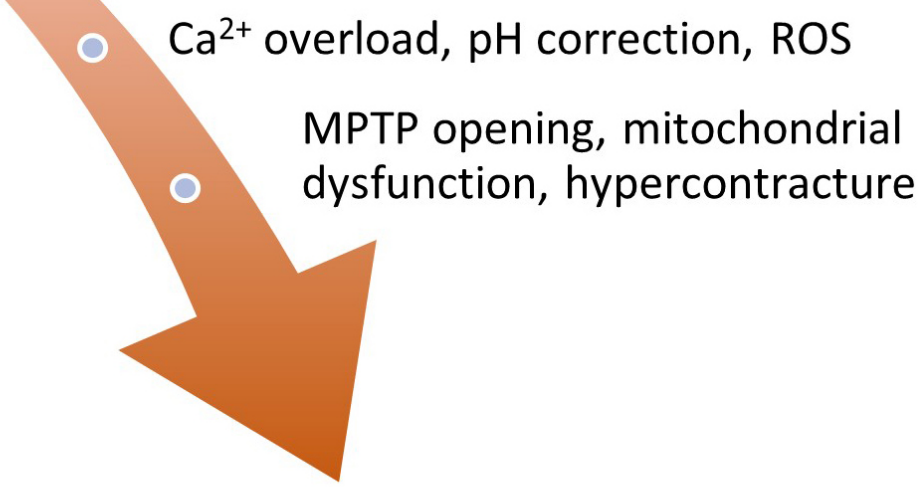

\section{CARDIOMYOCYTE DEATH}

Fig. 1. Proposed mechanisms of cardiomyocyte death during ischaemia-reperfusion. A diagrammatic overview of the processes involved during both the ischaemic, and reperfusion phases with oxidative stress leading to production of reactive oxygen species (ROS), mitochondrial calcium overload, and effects on the mitochondrial permeability transition pore (MPTP) resulting in cardiomyocyte death.

\section{Pathophysiology}

Acute coronary artery occlusion in ST-segment elevation myocardial infarction results in myocardial ischaemia downstream of the blockage. This initiates a cascade of abnormalities affecting the function, metabolism, and structure of myocytes, ultimately leading to necrosis and cell death [13]. Reimer and Jennings illustrated this in 1977 after work on myocardial ischaemia in the dog model, describing a "wavefront phenomenon", where irreversible injury of ischaemic myocardium occurs first in the subendocardium before extending to the subepicardial layers $[14,15]$. The resulting infarct size is determined by several key factors and is summarised in Fig. 1.

\subsection{Size of myocardial area at risk (AAR)}

The myocardial tissue in the vascular territory distal to the occlusion is at definite risk of ischaemic death if not reperfused in a timely fashion and is known as the myocardial area at risk [13]. In humans, the proportion of AAR that is irreversibly injured ranges from $0 \%$ (aborted infarction) up to $88 \%$ (infarction), and is dependent on the infarct artery, reperfusion time, collateral flow, reperfusion injury, and microvascular dysfunction $[13,16]$. Myocardial salvage describes the proportion of the AAR that survives, and is an important determinant of final infarct size.

Assessment of AAR can be difficult. In humans, a commonly used technique is single-photon emission to- mography but this requires radioisotope to be injected during occlusion, and before reperfusion [13]. Given the logistical issues, this technique has limited clinical applicability. More recently, cardiac magnetic resonance (CMR) has been used to assess AAR. T2-weighted imaging enhances oedema, which is present in ischaemic myocardium [1719]. However, T2 imaging can be prone to artifact and requires long breath-holds, with insufficient image quality in up to $62 \%$ of patients [20-22]. More recently, contrastenhanced cine (CE-SSFP) was compared to T2-weighted imaging in 166 participants involved in a substudy of the DANAMI-3 trial (Complete revascularisation versus treatment of the culprit lesion only in patients with ST-segment elevation myocardial infarction and multivessel disease). This showed good internal consistency in assessment of the AAR along with good inter-observer agreement of analysis of contrast enhanced cine with the authors suggesting that CE-cine can replace T2-weighted imaging for a more valid assessment of myocardial AAR [23].

\subsection{Duration of occlusion}

Cardiomyocyte death from ischaemia commences if this period is greater than 20 minutes, arising from the subendocardium, and progressing into the subepicardium [14]. Despite this, some $30-50 \%$ of myocardium in the AAR is still viable after $4-6 \mathrm{~h}$ of symptom onset [24], with evidence from thrombolysis data that reperfusion can 
limit infarct size and reduce mortality in patients presenting with STEMI up to $12 \mathrm{~h}$ post coronary occlusion [2528]. It is from this data that consensus guidelines, such as those from the European Society of Cardiology (ESC) recommend reperfusion therapy in all patients with symptoms of ischaemia and ST-segment elevation of $\leq 12 \mathrm{~h}$ [4]. In asymptomatic patients, or those without electrocardiographic evidence of ongoing ischaemia $12-48 \mathrm{~h}$ after symptom onset, there is some evidence of benefit of PPCI vs medical therapy alone, with a small randomised control trial (Beyond 12 Hours Reperfusion Alternative Evaluation, BRAVE-2 trial) of 347 patients showing improved myocardial salvage and 4-year survival $[25,29]$. In very late presenting STEMI (3-28 days), the Occluded Artery Trial showed no improvement in LV systolic function or prognostic benefit in patients undergoing percutaneous coronary intervention [30].

\subsection{Collateral coronary blood flow}

Collateral vessels connect one epicardial vascular territory to another, providing an alternative source of blood supply to myocardium, should one epicardial artery occlude [31,32]. The phase 1 thrombolysis in myocardial infarction (TIMI) trial demonstrated reduced creatine kinase (CK) enzyme release and improved LV ejection fraction at discharge in patients presenting with acute myocardial infarction (AMI) and failed reperfusion in the presence of coronary collaterals [33]. Furthermore, the presence of well-developed vs poorly grown collateral vessels has been shown to significantly reduce major adverse cardiac events (MACE), and even cardiac mortality at 10 years $[34,35]$.

\subsection{Coronary microvascular dysfunction and lethal reperfusion injury}

While reperfusion is necessary for salvaging ischaemic myocardium, the process also leads to reversible injury by way of myocardial stunning [36], CMD, and death of cardiomyocytes that were viable at the end of the index ischaemic event (lethal reperfusion injury).

The most severe end of this spectrum is the phenomenon of "no-reflow", where there is inadequate myocardial perfusion despite re-establishing infarct related artery patency, with no angiographic evidence of mechanical obstruction. No-reflow affects between $11 \%$ and $41 \%$ of patients undergoing PPCI and significantly diminishes the beneficial impact of reperfusion therapy, results in poor clinical and functional outcomes, and is an independent predictor of increased infarct size and 5-year mortality [3742].

The pathophysiology of no-reflow is complex and multifactorial. Mechanisms implicated include:

(1) Pre-existing microvascular dysfunction. This may be structural or functional and impairs coronary flow reserve, increasing susceptibility to ischaemic injury. Risk factors include age, abnormal insulin resistance and lipid metabolism and chronic inflammatory disease. In addition, pre-existing microvascular dysfunction is an independent predictor of adverse cardiac events [30,31].

(2) Distal micro-thromboembolism. Thrombus debris migrate distally during balloon dilatation/stenting. It has been shown that myocardial perfusion falls when embolic microspheres block $>50 \%$ of coronary capillaries in humans [43]. Several trials assessing impact of thrombectomy have been disappointing, further highlighting the complex and multifactorial nature of no-reflow $[44,45]$.

(3) Ischaemic injury. Endothelial cell damage and necrosis causes loss of vascular integrity, extravascular accumulation of fluid and blood cells, altered production of nitric oxide, and cell swelling [46,47].

(4) Lethal reperfusion injury. This is discussed in more detail below, but major contributory factors include infiltration of neutrophils and platelets, oxidative stress, and mitochondrial dysfunction. This can lead to intramyocardial haemorrhage, a predictor of infarct size and poor clinical outcomes $[11,48]$.

\subsection{Lethal reperfusion injury}

The timeframe for lethal reperfusion injury (RI) is difficult to accurately quantify. Clearly, a significant amount of damage takes place within the first few minutes of reperfusion, which has been demonstrated in both rat hearts and in humans [49-51]. However, it is difficult to distinguish between ischaemic cell death or lethal RI (or a combination of both) as the cause of apoptosis and late cell death [12]. Several important processes have been identified in experimental studies that act in concert leading to lethal RI.

(1) Oxidative stress. Myocardial hypoxia leads to dysfunction of the electron transport chain in the mitochondria, with decreased adenosine triphosphate production (ATP) and anaerobic metabolism. One of the subsequent consequences is decreased anti-oxidative intracellular agents [52]. During the first few minutes of reperfusion, reactive oxygen species (ROS) are generated by the xanthine oxidase and nicotinamide adenine dinucleotide phosphate oxidase systems, mitochondrial electron transport chain, and uncoupling of the nitric oxide synthase (NOS) system [5254] . ROS induce opening of the mitochondrial permeability transition pore (MPTP), act as a neutrophil chemoattractant, and lead to dysfunction of the sarcoplasmic reticulum. They also induce cell damage and ultimately cause cell death via autophagy (damaged components in the cytoplasm that are degraded in membrane vesicles), necrosis (unlike apoptosis, is passive and unregulated), and apoptosis (programmed cell death activated under hypoxic stress and in response to ROS) $[52,55,56]$.

(2) Intracellular and mitochondrial calcium overload. This starts during acute ischaemia and is exacerbated during reperfusion by ROS, disruption of the plasma membrane, and opening of the MPTP [57]. 
(3) Rapid restoration of physiological $\mathrm{pH}$. During reperfusion, intracellular myocardial $\mathrm{pH}$ rapidly normalises from less than 7.0 during acute ischaemia. This contributes to myocyte death by permitting MPTP opening and hypercontracture [58].

(4) Mitochondrial permeability transition pore. Many proposed mechanisms of ischaemia-reperfusion injury (IRI) converge on MPTP, a mitochondrial membrane channel. Rat heart models show that opening leads to membrane depolarisation and uncoupling of oxidative phosphorylation, leading to ATP depletion and cell death $[59,60]$. During IRI, MPTP remains closed during ischaemia, before opening at reperfusion in response to mitochondrial calcium and phosphate overload, ROS, and rapid $\mathrm{pH}$ correction [61].

\subsection{Extracellular vesicles (EVS)}

Blood contains large numbers of EVs that originate from a variety of cells, including platelets, erythrocytes, leucocytes, and endothelium [62-64]. These small vesicles aid intercellular communication during IRI by transferring their contents (lipids, amino-acids, proteins, mRNAs, miRNAs) [65]. There are different types of EVs.

In the past few years, there has been increasing interest in Exosomes, a lipid vesicle $50-150 \mathrm{~nm}$ in diameter that is secreted by all cells [63]. The exact mechanism of cardioprotection in IRI has yet to be fully unravelled but they have been shown to reduce scar size when injected into the infarct border, and improve cardiac function after AMI in mice [66]. Some exosomes contain short RNA, that have been shown to reduce infarct size $48 \mathrm{~h}$ after reperfusion in rats following $45 \mathrm{~min}$ of coronary artery occlusion [67].

Other EVs include microvesicles (MVs) which are involved in intercellular communication. Patients with heart failure have been found to have increasing circulating MVs, and they may also play a negative role in IRI, with MVs released from endothelial cells after ischaemicreperfusion acting as pro-apoptotic and pro-oxidative to cardiomyocytes [68]. More recently, EVs taken from patients presenting with acute coronary syndrome before PCI have been shown to protect against IRI, both in-vitro and in the rat heart [69].

\section{Cardioprotective strategies}

Prompt reperfusion in patients presenting with STEMI with PPCI remains the gold standard and improves outcomes. However, both experimental animal models and studies in patients with STEMI suggest that up to $50 \%$ of the final infarct size is a result of lethal reperfusion injury [11], making it an attractive therapeutic target (Fig. 2). Over the past 30 years, multiple cardioprotective strategies aimed at reducing myocardial IRI have been proposed, and although pre-clinical studies have often been promising, this has not translated into clinical efficacy [70]. Therefore, there remains an urgent need to discover novel therapies that can be given peri-procedurally to reduce infarct size and improve mortality and morbidity in reperfused STEMI patients. Numerous cardioprotective strategies have been proposed and tested, broadly divided into 3 treatment modalities (Fig. 3).

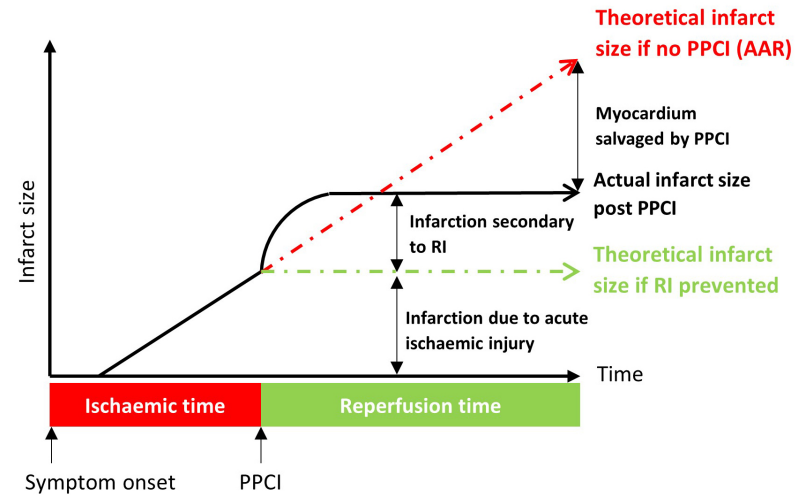

Fig. 2. This highlights the individual components that make up final myocardial infarct size (arbitrary value) post STEMI up to $24 \mathrm{~h}$ (black solid line). The dotted red line represents the theoretical infarct size if no PPCI (AAR - area at risk). The dotted green line represents the theoretical infarct size if $\mathrm{RI}$ is prevented. Figure modified from J Clinical Res [57].

\subsection{Ischaemic conditioning}

This involves brief cycles of ischaemia and reperfusion applied to an organ or tissue that may be pre, during, or post reperfusion; local or remote. The mechanisms are incompletely understood, but may involve changes to intracellular $\mathrm{pH}$, effects on nitric oxide synthases (NOS) and their subsequent generation of reactive oxygen and nitrogen species, along with increased protein kinase $\mathrm{G}$ and reperfusion injury salvage kinase [71].

Ischaemic post-conditioning (iPOST): alternating angioplasty balloon inflation/deflations immediately after opening the culprit artery has been shown to be protective in multiple Phase II trials [72,73]. However, the largest randomised control trial (RCT) to date (DANAMI-3, 2017) of 1234 patients presenting within $12 \mathrm{~h}$ of a STEMI with a thrombolysis in myocardial infarction (TIMI) flow of $0-1$ (absence or faint antegrade coronary flow beyond occlusion) showed that routine iPOST during PPCI failed to reduce the composite outcome of all cause death and hospitalisation for heart failure [74]. Limitations to iPOST include treatment being administered after reperfusion, thereby suggesting that reperfusion injury occurs before any potential benefit in this study protocol. The authors suggested a modified protocol involving post-conditioning immediately after reperfusion (e.g., within 15 seconds) [74].

Remote ischaemic conditioning (RIC): serial inflations and deflations with a pneumatic cuff on the arm or thigh to induce brief cycles of ischaemia and reperfusion showed promise in increasing myocardial salvage and re- 


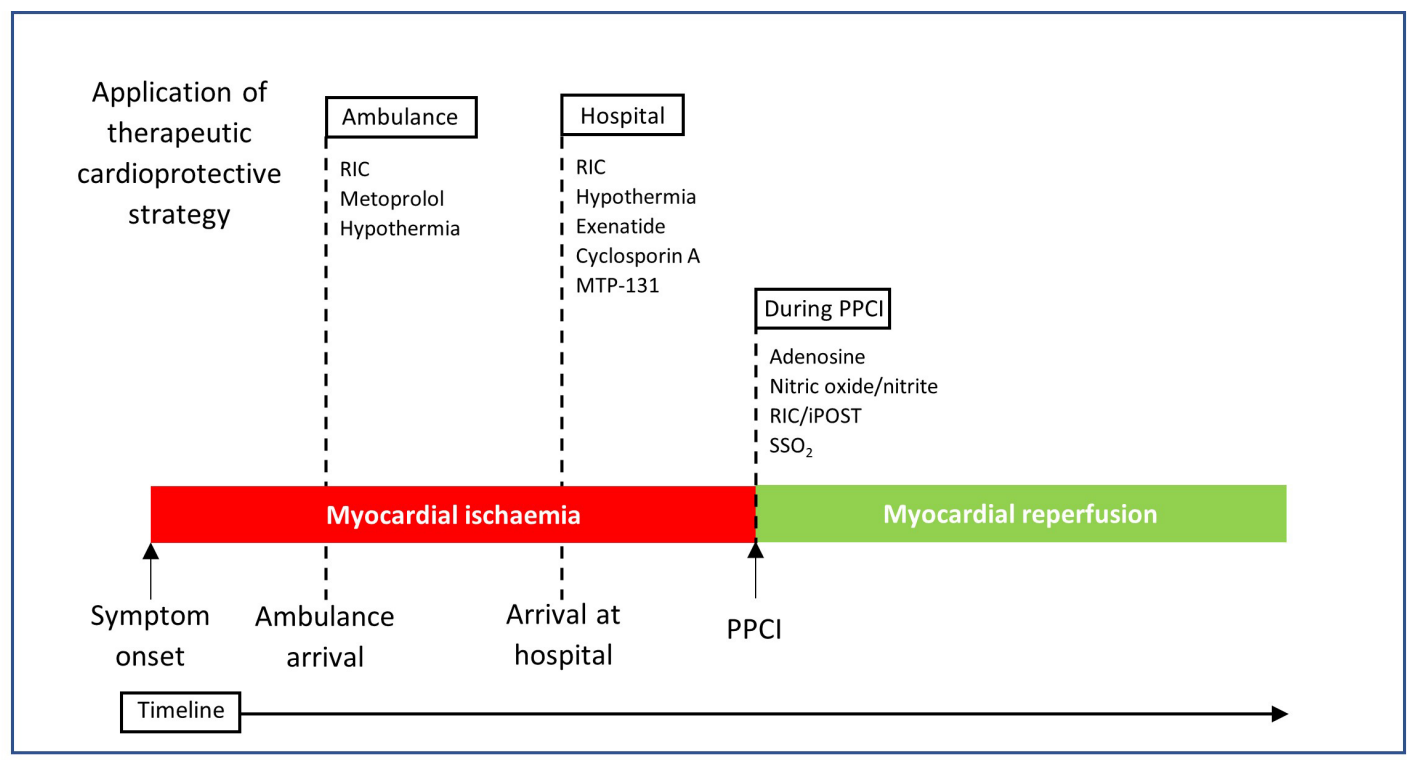

Fig. 3. Diagrammatic representation of the various timeframes of cardioprotective treatments designed to reduce MI size in STEMI patients. Treatment strategies may be attempted during the ischaemic phase, that is, prior to reperfusion with PPCI or thrombolysis, such as remote ischaemic conditioning (RIC), hypothermia, and some pharmacological therapies. They may also be given during myocardial reperfusion e.g., ischaemic post-conditioning (iPOST) and super saturated oxygen $\left(\mathrm{SSO}_{2}\right)$.

ducing infarct size by $20-30 \%$ in animal (dog) and small clinical human trials [75-78]. Despite these encouraging results, the largest RCT to date (CONDI-2/ERIC-PPCI, 2019) of 5401 patients presenting with STEMI failed to show improved clinical outcomes (cardiac death or hospitalisation for heart failure) at 12 months [79]. Several potential reasons for this have been proposed: (1) animal models used for IRI do not adequately represent a typical STEMI patient, (2) limb RIC protocol not sufficiently optimised for maximal cardioprotection, and (3) presence of co-morbidities that may confound results [80].

\subsection{Pharmacotherapy}

Multiple therapeutic agents have been shown to improve myocardial infarct size in animal/pre-clinical models. Here, we focus on those that have progressed to clinical trials.

Exenatide: this glucagon-like peptide-1 (GLP-1) receptor agonist has been shown in both small animal (rat) models and small clinical trials to significantly reduce myocardial infarct size, and increase myocardial salvage index $[81,82]$. The exact mechanism of cardio-protection is unclear but evidence suggests multiple modes of action, involving activation of phosphoinositide 3 kinase/cyclic adenosine monophosphate/cyclic guanosine monophosphate, inhibition of apoptotic factors, and attenuating the oxidative stress injury $[82,83]$. Further studies are required to assess for improved clinical outcomes.

Nitric oxide and nitrite: Two recent clinical studies have failed to demonstrate significant reduction in MI size with either intravenous, or intracoronary routes in STEMI patients treated with PPCI $[84,85]$. However, the latter did significantly reduce MACE at 1 year $(p=0.04)$ and infarct size in patients with STEMI and TIMI flow $\leq 1$ on both enzyme release and CMRI on subgroup analysis (19\% reduction in CK area under the curve (AUC), $p=0.03 ; 15.3 \%$ vs $20.08 \%$ infarct size on CMRI, $p=0.03$ ) [85].

Cyclosporin A: This inhibitor of MPTP showed promise in several small clinical trials, reducing CK AUC by $40 \%$, and infarct size by between $20-28 \%$ as assessed on CMR [86,87]. The Cyclosporine and Prognosis in AMI patients trial (CIRCUS, 2015), the largest to date involving 970 patients in patients with anterior STEMI showed no clinical benefit nor difference in CK, Troponin I release, and adverse left ventricular remodelling at 1 year when compared to placebo [88].

MTP-131: This peptide targets mitochondria, inhibiting cardiolipin, thereby reducing production of ROS. Once again, small animal (rat) models reported reduced MI size $[89,90]$, but in the Phase II 117 patient EMBRACE STEMI trial, no significant reduction in MI size was seen in a carefully selected population of patients presenting with an anterior STEMI, TIMI 0, and symptom onset time $<4 \mathrm{~h}$ [91].

Adenosine: There have been a total of 13 RCTs (4273 STEMI patients) assessing both intracoronary adenosine (8 RCTs) and intravenous adenosine (5 RCTs). A recent metaanalysis in 2015 found that patients who were administered intracoronary adenosine showed a significantly lower incidence of heart failure (risk ratio 0.44 [95\% confidence interval $0.25-0.78], p=0.005$ ) and coronary re-flow [92]. There was no difference in non-fatal MI or all-cause mortality. 
Metoprolol: IV metoprolol administered after 90 mins of coronary occlusion prior to reperfusion reduces MI size and improves LV systolic function in the porcine heart [93]. Results from clinical trials have been mixed. The proof-of-concept study (The Effect of Metoprolol in Cardioprotection During an Acute Myocardial Infarction (METOCARD-CNIC) trial) in 270 anterior STEMI patients given metoprolol as early as possible showed reduced MI size, preserved LV systolic function, and lowered hospital readmissions for heart failure [94].

However, the more recent phase III trial (the Early Intravenous Beta-Blockers in Patients with ST-Segment Elevation Myocardial Infarction Before PPCI (EARLY-BAMI) trial) in 683 patients in a non-restricted STEMI population showed effect on infarct size or LV function [95]. This difference may be in part due to the more select population in the METOCARD-CNIC trial (symptom onset $<6 \mathrm{~h}$, anterior STEMI) and with a lower dose of metoprolol given later post MI in EARLY-BAMI [96]. The importance of early treatment with metoprolol has been recently highlighted in the pig model, where intravenous (IV) metoprolol significantly reduced infarct size when given 30-50 mins after onset of coronary occlusion, but showed no effect when given after 20-25 mins, or after 60 mins occlusion [97]. This data can be interpreted in several ways, that metoprolol delays ischaemic damage rather than reducing lethal reperfusion injury, or that reperfusion injury is maximal at ischaemia of intermediate duration (30-50 mins) and therefore metoprolol has greatest effect here [98]. Unfortunately, there is currently no reliable method of distinguishing from ischaemic and lethal reperfusion injury [99].

Sodium thiosulphate (STS): this metabolite of hydrogen sulphide $\left(\mathrm{H}_{2} \mathrm{~S}\right)$, an endogenous gas transmitter, has been shown in mice models to mediate numerous physiological activities in organs such as the heart, brain, and kidney, by its anti-oxidant effects, preserving mitochondrial activity, and chelating calcium [100-102]. In vitro and murine studies have shown that STS can protect against neuronal ischaemia and IRI in isolated rat heart, significantly reducing infarct size $[103,104]$. These protective effects were associated with inhibition/reduced expression of caspase-3, a vital protein involved in cell apoptosis. Furthermore, in 2018, Ravindran et al. [105] showed isolated rat hearts treated with STS post-conditioning significantly reduced infarct size associated with RI by modulating cardiac mitochondria responsible for both contractile and metabolic function. IV STS is currently undergoing a phase 2 trial-Sodium Thiosulfate to Preserve Cardiac Function in STEMI (GIPS-IV).

\subsection{Physical intervention}

Therapeutic hyperoxaemia/super-saturated oxygen $\left(\mathrm{SSO}_{2}\right)$ : The Acute Myocardial Infarction with Hyperoxaemic Therapy (AMIHOT) I and II trials involved intracoronary hyperbaric hyperoxaemic infusion started after PPCI and continued for 90 mins [106,107]. While neither study showed a difference in 14-day MI size, the pooled results suggested a reduction in infarct size in patients with anterior STEMI presenting within $6 \mathrm{~h}$ of symptom onset. However, there were a numerically higher incidence of safety events. More recently, the Evaluation of Intracoronary Hyperoxaemic Oxygen Therapy in AMI patients (IC-HOT, 2019) study evaluated the safety of super-saturated Oxygen following PPCI via the left main stem in 100 patients without cardiogenic shock with a 60 -min $\mathrm{SSO}_{2}$ infusion and showed a favourable safety profile [108]. The follow-up 1-year outcomes were reported earlier this year showing that treatment with $\mathrm{SSO}_{2}$ was associated with a lower composite endpoint of all-cause death, new-onset heart failure (HF), or hospitalisation for HF [109]. While this was a modest, propensity-matched cohort study in selected patients, there is enough potential shown to warrant an appropriately powered RCT.

Therapeutic hypothermia: this has been consistently shown to reduce MI in animal models, and human preclinical studies [110]. A meta-analysis in 2016 included 6 RCTs, with a total of 819 patients found no significant reduction in all-cause mortality or heart failure [111]. More recently, both the Out-of-hospital initiation of hypothermia in STEMI trial and Cooling As an Adjunctive Therapy to Percutaneous Intervention in Patients with AMI EU (COOL AMI EU) showed no significant reduction in MI size, with the latter showing a significantly increased risk of paroxysmal atrial fibrillation and cardiogenic shock in the hypothermia group $[112,113]$.

\section{Discussion}

The accumulation of evidence described has shown numerous cardioprotective strategies that are effective at reducing infarct size in animal models. However, they have yet to translate into improvements in hard outcomes in clinical trials. In fact, many treatment modalities have shown early promise in small phase I/II trials but have fallen at the last hurdle when tested in large RCTs (in particular, iPOST and RIC). Many of the pharmacological therapies require larger studies to provide more definitive answers.

There are several possible reasons for these conflicting and often disappointing results.

\subsection{Inadequate animal models}

Often, pre-clinical studies are performed on small animals, such as mice, rats, and rabbits. Some potential treatments have not been studied in large animals. Those that have usually include healthy, young animals. This contrasts with the typical demographic of the STEMI patient who is older, with multiple co-morbidities, an on multiple medications that may interfere with the cardioprotective intervention, such as statins and platelet $\mathrm{P}_{2} \mathrm{Y}_{12}$ inhibitors $[114,115]$. In terms of size and anatomy of the heart and systemic haemodynamics, large animal models have an ad- 
vantage given that they are more like humans. Furthermore, large animals display a larger variation in haemodynamic responses and infarct size development, likely due to wider genetic variation [99].

\subsection{Inadequate study design}

The inclusion criteria should only include patients that have been shown to benefit most from adjunctive therapy to PPCI. This includes: a large AAR such as anterior STEMI, occluded culprit artery on presentation (TIMI $<1$ ), and no collaterals [57,116,117]. Outcome measures must also be carefully considered. In proof-of-concept studies, cardiac enzymes such as creatine kinase myocardial band and troponin may be used. However, more a more robust surrogate endpoint is infarct size (e.g., measured by CMR) and ultimately, clinical outcome measures such as mortality and heart failure must be determined [99].

\subsection{Dosing/intervention protocol}

Without good pre-clinical trials and pilot data, it may be difficult to establish effective treatment dosage or interventional protocol resulting in failure to show positive results in some studies. For example, the limb RIC protocol in the CONDI-2/ERIC-PPCI trial was not optimised for maximal cardioprotection (duration of limb ischaemia, and reperfusion cycles) which the authors note as one of the potential reasons why the trial failed to show a positive outcome [80].

\subsection{Route of pharmacotherapy}

The concentration of drug may vary significantly when given intravenous vs intracoronary. An example of this can be seen with the use of adenosine where intracoronary adenosine was associated with clinical improvement compared to intravenous injection [92].

\subsection{Timing of intervention}

There is evidence that intervention that is present at time of reperfusion can reduce MI size [118]. This may be difficult to apply in STEMI given the early administration required. Furthermore, treatment may not reach ischaemic myocardium in those with a completely occluded artery. Timing of intervention may be more complex than simply as soon as possible, as demonstrated with the use of IV metoprolol in the pig model, where no effect on infarct size was found if the coronary artery was occluded for too short ( $<25 \mathrm{mins}$ ), or too long ( $>60$ mins) a time [97].

\section{Future recommendations}

The pathophysiology of ischaemic RI is complex and multifactorial. Routinely used animal models may not adequately simulate this but are rather one element of it. It may be that a multi-pronged approach is required targeting the different players involved in IRI, or a therapy that acts on multiple pathways.
Clinical trials should be undertaken based on solid pre-clinical data, including those performed on large animals, with large RCTs only performed if Phase II trials have shown reduction in MI size. Furthermore, the trial design is crucial, and should ideally include patients with large AAR (e.g., anterior STEMI), TIMI $<1$ flow, and no collateral vessels.

\begin{abstract}
Abbreviations
AMI, acute myocardial infarction; IHD, ischaemic heart disease; IRI, ischaemia-reperfusion injury; MPTP, mitochondrial permeability transition pore; MVO, microvascular obstruction; STEMI, ST-segment elevation myocardial infarction; iPOST, ischaemic postconditioning; RIC, remote ischaemic conditioning; PPC, primary percutaneous coronary intervention; ROS, reactive oxygen species; AAR, area at risk.
\end{abstract}

\section{Author contributions}

The first draft of the manuscript was written by $\mathrm{JH}$ with supervision and contribution by WDS. NGB and AJL provided specialist expertise and advice regarding manuscript content and contributed to the final manuscript. WDS and ACS supervised the project. All authors read and approved the final manuscript.

\section{Ethics approval and consent to participate}

Not applicable.

\section{Acknowledgment}

We would like to express our gratitude to all those who helped us during the writing of this manuscript and for all the peer reviewers for their expert opinions and suggestions.

\section{Funding}

This research received no external funding.

\section{Conflict of interest}

The authors declare no conflict of interest.

\section{References}

[1] Townsend N, Wilson L, Bhatnagar P, Wickramasinghe K, Rayner M, Nichols M. Cardiovascular disease in Europe: epidemiological update 2016. European Heart Journal. 2016; 37 : 3232-3245.

[2] Lambert L, Brown K, Segal E, Brophy J, Rodes-Cabau J, Bogaty P. Association between timeliness of reperfusion therapy and clinical outcomes in ST-elevation myocardial infarction. The Journal of the American Medical Association. 2010; 303: 21482155.

[3] Berger PB, Ellis SG, Holmes DR, Granger CB, Criger DA, Betriu A, et al. Relationship between delay in performing direct coronary angioplasty and early clinical outcome in patients with acute myocardial infarction: results from the global use of strategies to open occluded arteries in Acute Coronary Syndromes (GUSTO-IIb) trial. Circulation. 1999; 100: 14-20. 
[4] Ibanez B, James S, Agewall S, Antunes MJ, Bucciarelli-Ducci C, Bueno H, et al. 2017 ESC Guidelines for the management of acute myocardial infarction in patients presenting with STsegment elevation: The Task Force for the management of acute myocardial infarction in patients presenting with ST-segment elevation of the European Society of Cardiology (ESC). European Heart Journal. 2018; 39: 119-177.

[5] Gibson CM, Schömig A. Coronary and myocardial angiography: angiographic assessment of both epicardial and myocardial perfusion. Circulation. 2004; 109: 3096-3105.

[6] Burns RJ, Gibbons RJ, Yi Q, Roberts RS, Miller TD, Schaer GL, et al. The relationships of left ventricular ejection fraction, endsystolic volume index and infarct size to six-month mortality after hospital discharge following myocardial infarction treated by thrombolysis. Journal of the American College of Cardiology. 2002; 39: 30-36.

[7] Wu E, Ortiz JT, Tejedor P, Lee DC, Bucciarelli-Ducci C, Kansal $\mathrm{P}$, et al. Infarct size by contrast enhanced cardiac magnetic resonance is a stronger predictor of outcomes than left ventricular ejection fraction or end-systolic volume index: prospective cohort study. Heart. 2008; 94: 730-736.

[8] Majidi M, Kosinski AS, Al-Khatib SM, Lemmert ME, Smolders $\mathrm{L}$, van Weert A, et al. Reperfusion ventricular arrhythmia 'bursts' predict larger infarct size despite TIMI 3 flow restoration with primary angioplasty for anterior ST-elevation myocardial infarction. European Heart Journal. 2009; 30: 757-764.

[9] Saia F, Grigioni F, Marzocchi A, Branzi A. Management of acute left ventricular dysfunction after primary percutaneous coronary intervention for ST elevation acute myocardial infarction. American Heart Journal. 2010; 160: S16-S21.

[10] Stone GW, Selker HP, Thiele H, Patel MR, Udelson JE, Ohman EM, et al. Relationship between Infarct Size and Outcomes Following Primary PCI. Journal of the American College of Cardiology. 2016; 67: 1674-1683.

[11] Yellon DM, Hausenloy DJ. Myocardial reperfusion injury. The New England Journal of Medicine. 2007; 357: 1121-1135.

[12] Piper HM, García-Dorado D, Ovize M. A fresh look at reperfusion injury. Cardiovascular Research. 1998; 38: 291-300.

[13] Rochitte CE, Azevedo CF. The myocardial area at risk. Heart. 2012; 98: 348-350.

[14] Reimer KA, Lowe JE, Rasmussen MM, Jennings RB. The wavefront phenomenon of ischemic cell death. 1. Myocardial infarct size vs duration of coronary occlusion in dogs. Circulation. 1977; 56: 786-794

[15] Reimer KA, Jennings RB. The 'wavefront phenomenon' of myocardial ischemic cell death. II. Transmural progression of necrosis within the framework of ischemic bed size (myocardium at risk) and collateral flow. Laboratory Investigation. 1979; 40: 633-644.

[16] Lee JT, Ideker RE, Reimer KA. Myocardial infarct size and location in relation to the coronary vascular bed at risk in man. Circulation. 1981; 64: 526-534.

[17] Kim RJ, Fieno DS, Parrish TB, Harris K, Chen EL, Simonetti O, et al. Relationship of MRI delayed contrast enhancement to irreversible injury, infarct age, and contractile function. Circulation. 1999; 100: 1992-2002.

[18] Arai AE, Leung S, Kellman P. Controversies in cardiovascular MR imaging: reasons why imaging myocardial T2 has clinical and pathophysiologic value in acute myocardial infarction. Radiology. 2012; 265: 23-32.

[19] Abdel-Aty H, Simonetti O, Friedrich MG. T2-weighted cardiovascular magnetic resonance imaging. Journal of Magnetic Resonance Imaging. 2007; 26: 452-459.

[20] Wince WB, Kim RJ. Molecular imaging: T2-weighted CMR of the area at risk-a risky business? Nature Reviews Cardiology. 2010; 7: 547-549.
[21] Lønborg J, Vejlstrup N, Mathiasen AB, Thomsen C, Jensen JS, Engstrøm T. Myocardial area at risk and salvage measured by T2-weighted cardiovascular magnetic resonance: reproducibility and comparison of two T2-weighted protocols. Journal of Cardiovascular Magnetic Resonance. 2011; 13: 50.

[22] Nordlund D, Klug G, Heiberg E, Koul S, Larsen TH, Hoffmann $\mathrm{P}$, et al. Multi-vendor, multicentre comparison of contrastenhanced SSFP and T2-STIR CMR for determining myocardium at risk in ST-elevation myocardial infarction. European Heart Journal Cardiovascular Imaging. 2016; 17: 744-753.

[23] Göransson C, Ahtarovski KA, Kyhl K, Lønborg J, NepperChristensen L, Bertelsen L, et al. Assessment of the myocardial area at risk: comparing $\mathrm{T} 2$-weighted cardiovascular magnetic resonance imaging with contrast-enhanced cine (CESSFP) imaging - a DANAMI3 substudy. European Heart Journal Cardiovascular Imaging. 2019; 20: 361-366.

[24] Ibáñez B, Heusch G, Ovize M, Van de Werf F. Evolving therapies for myocardial ischemia/reperfusion injury. Journal of the American College of Cardiology. 2015; 65: 1454-1471.

[25] Schömig A, Mehilli J, Antoniucci D, Ndrepepa G, Markwardt C, Di Pede F, et al. Mechanical reperfusion in patients with acute myocardial infarction presenting more than 12 hours from symptom onset: a randomized controlled trial. The Journal of the American Medical Association. 2005; 293: 2865-2872.

[26] Fibrinolytic Therapy Trialists' (FTT) Collaborative Group. Indications for fibrinolytic therapy in suspected acute myocardial infarction: collaborative overview of early mortality and major morbidity results from all randomised trials of more than 1000 patients. The Lancet. 1994; 343: 311-322.

[27] Alkhalil M, Choudhury RP. Reperfusion Treatment in Late Presentation Acute Myocardial Infarction: Timing Is Not Everything. Circulation: Cardiovascular Interventions. 2018; 11: e007287.

[28] Boersma E. Does time matter? A pooled analysis of randomized clinical trials comparing primary percutaneous coronary intervention and in-hospital fibrinolysis in acute myocardial infarction patients. European Heart Journal. 2006; 27: 779-788.

[29] Ndrepepa G. Mechanical Reperfusion and Long-term Mortality in Patients with Acute Myocardial Infarction Presenting 12 to 48 Hours from Onset of Symptoms. The Journal of the American Medical Association. 2009; 301: 487.

[30] Hochman JS, Lamas GA, Buller CE, Dzavik V, Reynolds HR, Abramsky SJ, et al. Coronary intervention for persistent occlusion after myocardial infarction. The New England Journal of Medicine. 2006; 355: 2395-2407.

[31] PITT B. Interarterial coronary anastomoses. Occurrence in normal hearts and in certain pathologic conditions. Circulation. 1959; 20: 816-822.

[32] Seiler C. The human coronary collateral circulation. European Journal of Clinical Investigation. 2010; 40: 465-476.

[33] Habib GB, Heibig J, Forman SA, Brown BG, Roberts R, Terrin $\mathrm{ML}$, et al. Influence of coronary collateral vessels on myocardial infarct size in humans. Results of phase I thrombolysis in myocardial infarction (TIMI) trial. The TIMI Investigators. Circulation. 1991; 83: 739-746.

[34] Billinger M, Kloos P, Eberli FR, Windecker S, Meier B, Seiler C. Physiologically assessed coronary collateral flow and adverse cardiac ischemic events: a follow-up study in 403 patients with coronary artery disease. Journal of the American College of Cardiology. 2002; 40: 1545-1550.

[35] Meier P, Gloekler S, Zbinden R, Beckh S, de Marchi SF, Zbinden S, et al. Beneficial effect of recruitable collaterals: a 10 -year follow-up study in patients with stable coronary artery disease undergoing quantitative collateral measurements. Circulation. 2007; 116: 975-983.

[36] Bolli R. Myocardial 'stunning' in man. Circulation. 1992; 86: 
$1671-1691$

[37] Ito H, Maruyama A, Iwakura K, Takiuchi S, Masuyama T, Hori $\mathrm{M}$, et al. Clinical implications of the 'no reflow' phenomenon. a predictor of complications and left ventricular remodeling in reperfused anterior wall myocardial infarction. Circulation. 1996; 93: 223-228.

[38] Taylor AJ, Al-Saadi N, Abdel-Aty H, Schulz-Menger J, Messroghli DR, Friedrich MG. Detection of acutely impaired microvascular reperfusion after infarct angioplasty with magnetic resonance imaging. Circulation. 2004; 109: 2080-2085.

[39] Harrison RW, Aggarwal A, Ou F, Klein LW, Rumsfeld JS, Roe MT, et al. Incidence and outcomes of no-reflow phenomenon during percutaneous coronary intervention among patients with acute myocardial infarction. The American Journal of Cardiology. 2013 ; 111: 178-184.

[40] Schwartz BG, Kloner RA. Coronary no reflow. Journal of Molecular and Cellular Cardiology. 2012; 52: 873-882.

[41] Resnic FS, Wainstein M, Lee MKY, Behrendt D, Wainstein RV, Ohno-Machado L, et al. No-reflow is an independent predictor of death and myocardial infarction after percutaneous coronary intervention. American Heart Journal. 2003; 145: 42-46.

[42] Ndrepepa G, Tiroch K, Fusaro M, Keta D, Seyfarth M, Byrne $\mathrm{RA}$, et al. 5-year prognostic value of no-reflow phenomenon after percutaneous coronary intervention in patients with acute myocardial infarction. Journal of the American College of Cardiology. 2010; 55: 2383-2389.

[43] Niccoli G, Scalone G, Lerman A, Crea F. Coronary microvascular obstruction in acute myocardial infarction. European Heart Journal. 2016; 37: 1024-1033.

[44] Fröbert O, Lagerqvist B, Olivecrona GK, Omerovic E, Gudnason T, Maeng M, et al. Thrombus Aspiration during STSegment Elevation Myocardial Infarction. New England Journal of Medicine. 2013; 369: 1587-1597.

[45] Jolly SS, James S, Džavík V, Cairns JA, Mahmoud KD, Zijlstra F, et al. Thrombus Aspiration in ST-Segment-Elevation Myocardial Infarction: an Individual Patient Meta-Analysis: Thrombectomy Trialists Collaboration. Circulation. 2017; 135: 143-152.

[46] Tranum-Jensen J, Janse MJ, Fiolet WT, Krieger WJ, D'Alnoncourt CN, Durrer D. Tissue osmolality, cell swelling, and reperfusion in acute regional myocardial ischemia in the isolated porcine heart. Circulation Research. 1981; 49: 364-381.

[47] Durante A, Camici PG. Novel insights into an "old" phenomenon: the no reflow. International Journal of Cardiology. 2015; 187: 273-280.

[48] Jelinek M, Santamaria J. Metamorphosis: the natural history of coronary heart disease. Sudden death is common. Unexpected death is not. International Journal of Cardiology. 2007; 118: 1013.

[49] Garcia-Dorado D, Ruiz-Meana M, Piper HM. Lethal reperfusion injury in acute myocardial infarction: facts and unresolved issues. Cardiovascular Research. 2009; 83: 165-168.

[50] Kin H, Zhao Z, Sun H, Wang N, Corvera JS, Halkos ME, et al. Postconditioning attenuates myocardial ischemia-reperfusion injury by inhibiting events in the early minutes of reperfusion. Cardiovascular Research. 2004; 62: 74-85.

[51] Inserte J, Barba I, Hernando V, Garcia-Dorado D. Delayed recovery of intracellular acidosis during reperfusion prevents calpain activation and determines protection in postconditioned myocardium. Cardiovascular Research. 2009; 81: 116-122.

[52] Wu M, Yiang G, Liao W, Tsai AP, Cheng Y, Cheng P, et al. Current Mechanistic Concepts in Ischemia and Reperfusion Injury. Cellular Physiology and Biochemistry. 2018; 46: 1650-1667.

[53] Zweier JL, Flaherty JT, Weisfeldt ML. Direct measurement of free radical generation following reperfusion of ischemic my- ocardium. Proceedings of the National Academy of Sciences of the United States of America. 1987; 84: 1404-1407.

[54] Hearse DJ, Humphrey SM, Chain EB. Abrupt reoxygenation of the anoxic potassium-arrested perfused rat heart: a study of myocardial enzyme release. Journal of Molecular and Cellular Cardiology. 1973; 5: 395-407.

[55] Ma S, Wang Y, Chen Y, Cao F. The role of the autophagy in myocardial ischemia/reperfusion injury. Biochimica Et Biophysica Acta. 2015; 1852: 271-276.

[56] Kalogeris T, Bao Y, Korthuis RJ. Mitochondrial reactive oxygen species: a double edged sword in ischemia/reperfusion vs preconditioning. Redox Biology. 2014; 2: 702-714.

[57] Hausenloy DJ, Yellon DM. Myocardial ischemia-reperfusion injury: a neglected therapeutic target. The Journal of Clinical Investigation. 2013; 123: 92-100.

[58] Lemasters JJ, Bond JM, Chacon E, Harper IS, Kaplan SH, Ohata $\mathrm{H}$, et al. The $\mathrm{pH}$ paradox in ischemia-reperfusion injury to cardiac myocytes. Experientia Supplementum. 1996; 76: 99-114.

[59] Hausenloy DJ, Yellon DM. The mitochondrial permeability transition pore: its fundamental role in mediating cell death during ischaemia and reperfusion. Journal of Molecular and Cellular Cardiology. 2003; 35: 339-341.

[60] Heusch G, Boengler K, Schulz R. Inhibition of mitochondrial permeability transition pore opening: the Holy Grail of cardioprotection. Basic Research in Cardiology. 2010; 105: 151-154.

[61] Griffiths EJ, Halestrap AP. Mitochondrial non-specific pores remain closed during cardiac ischaemia, but open upon reperfusion. Biochemical Journal. 1995; 307: 93-98.

[62] Boulanger CM, Loyer X, Rautou P, Amabile N. Extracellular vesicles in coronary artery disease. Nature Reviews Cardiology. 2017; 14: 259-272.

[63] Davidson SM, Yellon DM. Exosomes and cardioprotection A critical analysis. Molecular Aspects of Medicine. 2018; 60: 104-114.

[64] Lawson C, Vicencio JM, Yellon DM, Davidson SM. Microvesicles and exosomes: new players in metabolic and cardiovascular disease. The Journal of Endocrinology. 2016; 228: R57-R71.

[65] Barani B, Rajasingh S, Rajasingh J. Exosomes: Outlook for Future Cell-Free Cardiovascular Disease Therapy. Exosomes in Cardiovascular Diseases. 2017; 123: 285-307.

[66] Barile L, Lionetti V, Cervio E, Matteucci M, Gherghiceanu M, Popescu LM, et al. Extracellular vesicles from human cardiac progenitor cells inhibit cardiomyocyte apoptosis and improve cardiac function after myocardial infarction. Cardiovascular Research. 2014; 103: 530-541.

[67] de Couto G, Gallet R, Cambier L, Jaghatspanyan E, Makkar $\mathrm{N}$, Dawkins JF, et al. Exosomal MicroRNA Transfer into Macrophages Mediates Cellular Postconditioning. Circulation. 2017; 136: 200-214.

[68] Zhang Q, Shang M, Zhang M, Wang Y, Chen Y, Wu Y, et al. Microvesicles derived from hypoxia/reoxygenation-treated human umbilical vein endothelial cells promote apoptosis and oxidative stress in H9c2 cardiomyocytes. BMC Cell Biology. 2016; 17: 25 .

[69] D'Ascenzo F, Femminò S, Ravera F, Angelini F, Caccioppo A, Franchin L, et al. Extracellular vesicles from patients with Acute Coronary Syndrome impact on ischemia-reperfusion injury. Pharmacological Research. 2021; 170: 105715.

[70] Hausenloy DJ, Garcia-Dorado D, Bøtker HE, Davidson SM, Downey J, Engel FB, et al. Novel targets and future strategies for acute cardioprotection: Position Paper of the European Society of Cardiology Working Group on Cellular Biology of the Heart. Cardiovascular Research. 2017; 113: 564-585.

[71] Davidson SM, Ferdinandy P, Andreadou I, Bøtker HE, Heusch G, Ibáñez B, et al. Multitarget Strategies to Reduce Myocardial Ischemia/Reperfusion Injury: JACC Review Topic of the Week. 
Journal of the American College of Cardiology. 2019; 73: 8999.

[72] Thibault H, Piot C, Staat P, Bontemps L, Sportouch C, Rioufol $\mathrm{G}$, et al. Long-Term Benefit of Postconditioning. Circulation. 2008; 117: 1037-1044.

[73] Staat P, Rioufol G, Piot C, Cottin Y, Cung TT, L'Huillier I, et al. Postconditioning the human heart. Circulation. 2005; 112: 2143-2148.

[74] Engstrøm T, Kelbæk H, Helqvist S, Høfsten DE, Kløvgaard L, Clemmensen $\mathrm{P}$, et al. Effect of Ischemic Postconditioning during Primary Percutaneous Coronary Intervention for Patients with ST-Segment Elevation Myocardial Infarction: a Randomized Clinical Trial. JAMA Cardiology. 2017; 2: 490-497.

[75] Przyklenk K, Bauer B, Ovize M, Kloner RA, Whittaker P. Regional ischemic 'preconditioning' protects remote virgin myocardium from subsequent sustained coronary occlusion. Circulation. 1993; 87: 893-899.

[76] White SK, Frohlich GM, Sado DM, Maestrini V, Fontana M, Treibel TA, et al. Remote Ischemic Conditioning Reduces Myocardial Infarct Size and Edema in Patients with ST-Segment Elevation Myocardial Infarction. JACC: Cardiovascular Interventions. 2015; 8: 178-188.

[77] Crimi G, Pica S, Raineri C, Bramucci E, De Ferrari GM, Klersy $\mathrm{C}$, et al. Remote ischemic post-conditioning of the lower limb during primary percutaneous coronary intervention safely reduces enzymatic infarct size in anterior myocardial infarction: a randomized controlled trial. JACC: Cardiovascular Interventions. 2013; 6: 1055-1063.

[78] Bøtker HE, Kharbanda R, Schmidt MR, Bøttcher M, Kaltoft AK, Terkelsen CJ, et al. Remote ischaemic conditioning before hospital admission, as a complement to angioplasty, and effect on myocardial salvage in patients with acute myocardial infarction: a randomised trial. The Lancet. 2010; 375: 727-734.

[79] Hausenloy DJ, Kharbanda RK, Møller UK, Ramlall M, Aarøe $\mathrm{J}$, Butler R, et al. Effect of remote ischaemic conditioning on clinical outcomes in patients with acute myocardial infarction (CONDI-2/ERIC-PPCI): a single-blind randomised controlled trial. The Lancet. 2019; 394: 1415-1424.

[80] Hausenloy DJ, Ntsekhe M, Yellon DM. A future for remote ischaemic conditioning in high-risk patients. Basic Research in Cardiology. 2020; 115: 35.

[81] Sonne DP, Engstrøm T, Treiman M. Protective effects of GLP-1 analogues exendin-4 and GLP-1(9-36) amide against ischemiareperfusion injury in rat heart. Regulatory Peptides. 2008; 146 : 243-249.

[82] Lønborg J, Vejlstrup N, Kelbæk H, Bøtker HE, Kim WY, Mathiasen $\mathrm{AB}$, et al. Exenatide reduces reperfusion injury in patients with ST-segment elevation myocardial infarction. European Heart Journal. 2012; 33: 1491-1499.

[83] Zhang Y, Qian P, Zhou H, Shen R, Hu B, Shen Y, et al. Pharmacological Signatures of the Exenatide Nanoparticles Complex against Myocardial Ischemia Reperfusion Injury. Kidney and Blood Pressure Research. 2018; 43: 1273-1284.

[84] Siddiqi N, Neil C, Bruce M, MacLennan G, Cotton S, Papadopoulou $\mathrm{S}$, et al. Intravenous sodium nitrite in acute STelevation myocardial infarction: a randomized controlled trial (NIAMI). European Heart Journal. 2014; 35: 1255-1262.

[85] Jones DA, Pellaton C, Velmurugan S, Rathod KS, Andiapen M, Antoniou S, et al. Randomized Phase 2 Trial of Intracoronary Nitrite during Acute Myocardial Infarction. Circulation Research. 2015; 116: 437-447.

[86] Piot C, Croisille P, Staat P, Thibault H, Rioufol G, Mewton N, et al. Effect of cyclosporine on reperfusion injury in acute myocardial infarction. The New England Journal of Medicine. 2008; 359: 473-481.

[87] Mewton N, Croisille P, Gahide G, Rioufol G, Bonnefoy E,
Sanchez I, et al. Effect of cyclosporine on left ventricular remodeling after reperfused myocardial infarction. Journal of the American College of Cardiology. 2010; 55: 1200-1205.

[88] Cung T, Morel O, Cayla G, Rioufol G, Garcia-Dorado D, Angoulvant $\mathrm{D}$, et al. Cyclosporine before PCI in Patients with Acute Myocardial Infarction. The New England Journal of Medicine. 2015; 373: 1021-1031.

[89] Dai W, Shi J, Gupta RC, Sabbah HN, Hale SL, Kloner RA. Bendavia, a mitochondria-targeting peptide, improves postinfarction cardiac function, prevents adverse left ventricular remodeling, and restores mitochondria-related gene expression in rats. Journal of Cardiovascular Pharmacology. 2014; 64: 543-553.

[90] Shi J, Dai W, Hale SL, Brown DA, Wang M, Han X, et al. Bendavia restores mitochondrial energy metabolism gene expression and suppresses cardiac fibrosis in the border zone of the infarcted heart. Life Sciences. 2015; 141: 170-178.

[91] Gibson CM, Giugliano RP, Kloner RA, Bode C, Tendera M, Jánosi A, et al. EMBRACE STEMI study: a Phase 2a trial to evaluate the safety, tolerability, and efficacy of intravenous MTP-131 on reperfusion injury in patients undergoing primary percutaneous coronary intervention. European Heart Journal. 2016; 37: 1296-1303.

[92] Bulluck H, Sirker A, Loke YK, Garcia-Dorado D, Hausenloy DJ. Clinical benefit of adenosine as an adjunct to reperfusion in ST-elevation myocardial infarction patients: an updated metaanalysis of randomized controlled trials. International Journal of Cardiology. 2016; 202: 228-237.

[93] Ibanez B, Prat-González S, Speidl WS, Vilahur G, Pinero A, Cimmino G, et al. Early Metoprolol Administration before Coronary Reperfusion Results in Increased Myocardial Salvage: analysis of ischemic myocardium at risk using cardiac magnetic resonance. Circulation. 2007; 115: 2909-2916.

[94] Ibanez B, Macaya C, Sánchez-Brunete V, Pizarro G, FernándezFriera L, Mateos A, et al. Effect of early metoprolol on infarct size in ST-segment-elevation myocardial infarction patients undergoing primary percutaneous coronary intervention: the Effect of Metoprolol in Cardioprotection during an Acute Myocardial Infarction (METOCARD-CNIC) trial. Circulation. 2013; 128: $1495-1503$.

[95] Roolvink V, Ibáñez B, Ottervanger JP, Pizarro G, van Royen $\mathrm{N}$, Mateos A, et al. Early Intravenous Beta-Blockers in Patients With ST-Segment Elevation Myocardial Infarction Before Primary Percutaneous Coronary Intervention. Journal of the American College of Cardiology. 2016; 67: 2705-2715.

[96] Hausenloy DJ, Barrabes JA, Bøtker HE, Davidson SM, Di Lisa $\mathrm{F}$, Downey $\mathrm{J}$, et al. Ischaemic conditioning and targeting reperfusion injury: a 30 year voyage of discovery. Basic Research in Cardiology. 2016; 111: 70 .

[97] Lobo-Gonzalez M, Galán-Arriola C, Rossello X González-Del-Hoyo M, Vilchez JP, Higuero-Verdejo MI, et al. Metoprolol blunts the time-dependent progression of infarct size. Basic Research in Cardiology. 2020; 115: 55.

[98] Kleinbongard P. Cardioprotection by early metoprolol- attenuation of ischemic vs. reperfusion injury? Basic Research in Cardiology. 2020; 115: 54.

[99] Bøtker HE, Hausenloy D, Andreadou I, Antonucci S, Boengler $\mathrm{K}$, Davidson SM, et al. Practical guidelines for rigor and reproducibility in preclinical and clinical studies on cardioprotection. Basic Research in Cardiology. 2018; 113: 39.

[100] Kim H, Kim H, Lee K, Kim J, Kim HS, Kim J, et al. A-Lipoic acid attenuates vascular calcification via reversal of mitochondrial function and restoration of Gas6/Axl/Akt survival pathway. Journal of Cellular and Molecular Medicine. 2012; 16: 273-286.

[101] Sen U, Vacek TP, Hughes WM, Kumar M, Moshal KS, Tyagi $\mathrm{N}$, et al. Cardioprotective role of sodium thiosulfate on chronic heart failure by modulating endogenous $\mathrm{H} 2 \mathrm{~S}$ generation. Phar- 
macology. 2008; 82: 201-213.

[102] Araya CE, Fennell RS, Neiberger RE, Dharnidharka VR. Sodium thiosulfate treatment for calcific uremic arteriolopathy in children and young adults. Clinical Journal of the American Society of Nephrology. 2006; 1: 1161-1166.

[103] Marutani E, Yamada M, Ida T, Tokuda K, Ikeda K, Kai S, et al. Thiosulfate Mediates Cytoprotective Effects of Hydrogen Sulfide against Neuronal Ischemia. Journal of the American Heart Association. 2015; 4: e002125

[104] Ravindran S, Jahir Hussain S, Boovarahan SR, Kurian GA. Sodium thiosulfate post-conditioning protects rat hearts against ischemia reperfusion injury via reduction of apoptosis and oxidative stress. Chemico-Biological Interactions. 2017; 274: 24 34.

[105] Ravindran S, Kurian GA. Effect of Sodium Thiosulfate Postconditioning on Ischemia-Reperfusion Injury Induced Mitochondrial Dysfunction in Rat Heart. Journal of Cardiovascular Translational Research. 2018; 11: 246-258.

[106] O’Neill WW, Martin JL, Dixon SR, Bartorelli AL, Trabattoni D, Oemrawsingh PV, et al. Acute Myocardial Infarction with Hyperoxemic Therapy (AMIHOT): a prospective, randomized trial of intracoronary hyperoxemic reperfusion after percutaneous coronary intervention. Journal of the American College of Cardiology. 2007; 50: 397-405.

[107] Stone GW, Martin JL, de Boer M, Margheri M, Bramucci E, Blankenship JC, et al. Effect of supersaturated oxygen delivery on infarct size after percutaneous coronary intervention in acute myocardial infarction. Circulation: Cardiovascular Interventions. 2009; 2: 366-375.

[108] David SW, Khan ZA, Patel NC, Metzger DC, Wood FO, Wasserman HS, et al. Evaluation of intracoronary hyperoxemic oxygen therapy in acute anterior myocardial infarction: the IC-HOT study. Catheterization and Cardiovascular Interventions. 2019; 93: 882-890.

[109] Chen S, David SW, Khan ZA, Metzger DC, Wasserman HS, Lotfi AS, et al. One-year outcomes of supersaturated oxygen therapy in acute anterior myocardial infarction: the IC-HOT study. Catheterization and Cardiovascular Interventions. 2021; 97: 1120-1126.
[110] Herring MJ, Hale SL, Dai W, Oskui PM, Kloner RA. Hypothermia in the setting of experimental acute myocardial infarction: a comprehensive review. Therapeutic Hypothermia and Temperature Management. 2014; 4: 159-167.

[111] Villablanca PA, Rao G, Briceno DF, Lombardo M, Ramakrishna H, Bortnick A, et al. Therapeutic hypothermia in ST elevation myocardial infarction: a systematic review and metaanalysis of randomised control trials. Heart. 2016; 102: 712 719.

[112] Testori C, Beitzke D, Mangold A, Sterz F, Loewe C, Weiser C, et al. Out-of-hospital initiation of hypothermia in ST-segment elevation myocardial infarction: a randomised trial. Heart. 2019; 105: 531-537.

[113] Noc M, Laanmets P, Neskovic AN, Petrović M, Stanetic B, Aradi D, et al. A multicentre, prospective, randomised controlled trial to assess the safety and effectiveness of cooling as an adjunctive therapy to percutaneous intervention in patients with acute myocardial infarction: the COOL AMI EU Pivotal Trial. EuroIntervention. 2021; 17: 466-473.

[114] Ferdinandy P, Hausenloy DJ, Heusch G, Baxter GF, Schulz $\mathrm{R}$. Interaction of risk factors, comorbidities, and comedications with ischemia/reperfusion injury and cardioprotection by preconditioning, postconditioning, and remote conditioning. Pharmacological Reviews. 2014; 66: 1142-1174.

[115] Schulz R, Andreadou I, Hausenloy DJ, Ferdinandy P. Risk factors, co-morbidities, and co-medications in cardioprotection: Importance for translation. British Journal of Pharmacology. 2020; 177: 5249-5251.

[116] Roubille F, Mewton N, Elbaz M, Roth O, Prunier F, Cung TT, et al. No post-conditioning in the human heart with thrombolysis in myocardial infarction flow 2-3 on admission. European Heart Journal. 2014; 35: 1675-1682.

[117] Schwartz Longacre L, Kloner RA, Arai AE, Baines CP, Bolli $\mathrm{R}$, Braunwald E, et al. New Horizons in Cardioprotection. Circulation: recommendations from the 2010 National Heart, Lung, and Blood Institute Workshop. 2011; 124: 1172-1179.

[118] Hausenloy DJ, Botker HE, Engstrom T, Erlinge D, Heusch G, Ibanez B, et al. Targeting reperfusion injury in patients with STsegment elevation myocardial infarction: trials and tribulations. European Heart Journal. 2017; 38: 935-941. 